\title{
Plasma Astrophysics in Laboratory
}

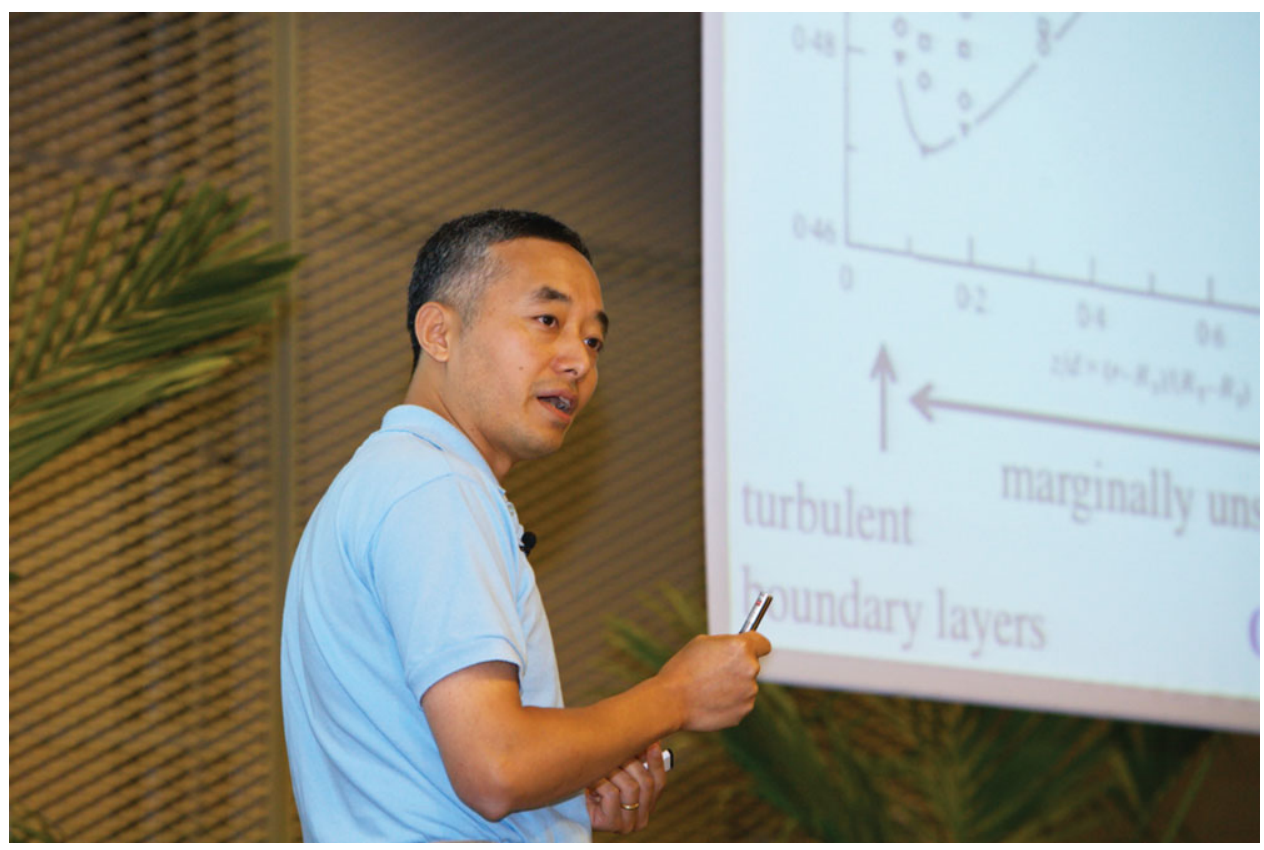

Hantao Ji 


\title{
The magnetized universe: its origin and dissipation through acceleration and leakage to the voids
}

\author{
Stirling A. Colgate ${ }^{1}$, Hui Li $^{2}$, Philipp P. Kronberg ${ }^{3}$ \\ ${ }^{1}$ MS B227, Los Alamos Nat. Lab., Los Alamos, N.M., 87545, USA \\ email: colgate@lanl.gov \\ ${ }^{2}$ MS B227, Los Alamos Nat. Lab., Los Alamos, N.M., 87545, USA \\ email: hli@lanl.gov \\ ${ }^{3}$ MS T006, Los Alamos Nat. Lab., Los Alamos, N.M., 87545, USA \\ email: kronberg@lanl.gov
}

\begin{abstract}
The consistency is awesome between over a dozen observations and the paradigm of radio lobes being immense sources of magnetic energy, flux, and relativistic electrons, - a magnetized universe.

The greater the total energy of an astrophysical phenomenon, the more restricted are the possible explanations. Magnetic energy is the most challenging because its origin is still considered problematic. We suggest that it is evident that the universe is magnetized because of radio lobes, ultra relativistic electrons, Faraday rotation measures, the polarized emission of extra galactic radio structures, the x-rays from relativistic electrons Comptonized on the CMB, and possibly extra galactic cosmic rays. The implied energies are so large that only the formation of supermassive black hole, (SMBH) at the center of every galaxy is remotely energetic enough to supply this immense energy, $\sim(1 / 10) 10^{8} M_{\odot} c^{2}$ per galaxy. Only a galaxy cluster of 1000 galaxies has comparable energy, but it is inversely, (to the number of galaxies), rare per galaxy. Yet this energy appears to be shared between magnetic fields and accelerated relativistic particles, both electrons and ions. Only a large-scale coherent dynamo generating poloidal flux within the accretion disk forming the massive black hole makes a reasonable starting point. The subsequent winding of this dynamo-derived magnetic flux by conducting, angular momentum-dominated accreting matter, $\left(\sim 10^{11}\right.$ turns near the event horizon in $10^{8}$ years $)$ produces the immense, coherent magnetic jets or total flux of radio lobes and similarly in star formation. By extending this same physics to supernova-neutron star formation, we predict that similar differential winding of the flux that couples explosion ejecta and a newly formed, rapidly rotating neutron star will produce similar phenomena of a magnetic jet and lobes in the forming supernova nebula. In all cases the conversion of force-free magnetic energy into accelerated ions and electrons is a major challenge.
\end{abstract}

Keywords. Magnetic fields, MHD, galaxies: jets, acceleration of particles.

\section{Introduction}

This talk is concerned with the magnetic fields in the universe: AGN, radio lobes, jets, star formation jets, galactic fields, stellar dynamos, and galaxy cluster dynamos. We believe that all these phenomena depend upon the same physics and we believe have a physically related explanation.

1) Observations: Radio Lobes, magnetized Jets, (synchrotron emission and its polarization), CRs and UHECRs, Faraday rotation, and x-rays. 
2) Energy; The magnetic energy: in radio lobes from luminosity and time is: $\sim 10^{8} M_{\odot} c^{2} / 10 \sim 10^{61}$ ergs. This energy is derived from mass accretion forming the $\mathrm{SMBH}$. A minimum energy requires that the magnetic energy equals the relativistic electron energy in the lobes.

3) The necesary large scale poloidal flux, requires a large scale magnetic dynamo in the accretion disk: (Despite good theoretical underpinning, three experiments have failed.)

We are (still) trying to demonstrate by experiment that two naturally occurring, orthogonal coherent motions, $\alpha$ and $\Omega$ will lead to a coherent dynamo that transforms a very small fraction, $\sim 10^{-11}$, of the free energy of accretion within an AGN disk, into poloidal magnetic energy within the disk. In addition and comparably important a small, $\sim 10^{-2} \alpha$-viscosity that mediates a fraction of the angular momentum transport within the disk, presumably by MRI or Rossby instabilities, is mediated by a weak turbulence and hence, leads to a small, necessary and finite magnetic flux diffusion. Around AGN supermassive BH's this is a Keplerian disk with differential winding, the $\Omega$ motion, and star-disk-collision-driven plumes for the helicity motion. It is only the finite mass of the disk, when driven as a plume several scale heights above the disk that after expansion has enough moment of inertia so that it can twist toroidal to poloidal field. (Magnetic buoyancy leads to magnetic loops that are largely devoid of mass and so do not have enough inertia to create the necessary torque.) In stellar dynamos the same coherent dynamo most likely occurs where the entropy gradient stabilizes rotational shear at the base of the convective zone, the $\Omega$ motion, and semi-coherent scale height-size, convective plumes occur at the base of the convective zone above the stable shear, producing the $\alpha$-helicity. We believe that the role of turbulence is not the conversion of kinetic to magnetic energy, but has the equally important function of allowing the magnetic flux to diffuse and self organize, between semi-coherent, otherwise perfectly conducting, orthogonal motions.

2) The production of a still larger, $\sim \times 10^{11}$, magnetic energy and magnetic flux by winding or twisting of this poloidal flux, external to the disk in a conducting medium, by the angular momentum flux of the disk itself.

Immense toroidal flux is produced by the differential winding of the AGN dynamogenerated poloidal flux by $N_{\text {turns }} \simeq 3 \times 10^{11}$ turns at the event horizon radius, $r_{g}$, in $10^{8}$ years, (the AGN accretion time at the Eddington limit and the radio lobe life time). The foot-prints of poloidal flux are attached by conduction to different radii of the accretion disk and so the differential winding of the foot prints at different radii leads to the force-free helical jet.

At a much smaller scale, but still awesomely large, the collapse of a type II supernova should lead similarly to large differential winding of the flux trapped by conduction between the neutron star and the ejecta. The neutron star will make $\sim 5 \times 10^{9}$ turns at $\sim 500 \mathrm{~Hz}$ during the spin-down time of $\sim 1 / 3$ year with the flux trapped in a conducting medium. The result of this immense winding in a conducting medium, can be seen as a nested set of Force-Free cylindrical helical magnetic flux surfaces, a pinch in plasma physics or a jet when seen in radio emission and made visible by the emission or selfluminosity of the parallel current carriers supporting the Force-Free helical magnetic fields, (similar to laboratory pinch or tokamak experiments) - - and leading to the supernova remnant emissions observed in the radio, optical, $\mathrm{x}$-rays, gamma rays, and cosmic rays.

3) In galaxies, a galactic -scale dynamo may exist, and because of a small, but finite number of turns in a Hubble time, probably serves solely to maintain a previously trapped flux originating from the AGN's helical magnetic jet.

The galaxy-scale field may be maintained, not generated, at just the back reaction limit where the galactic in-fall pressure from gas accretion (a galactic gas mass, $10 \%$, 
in a Hubble time), from the galaxy halo onto the galaxy just balances the magnetic and cosmic ray pressure within the galaxy. This "seed" field flux is probably trapped during galaxy formation from the jet, the Force-Free helix, from the AGN dynamo and disk. It is a small, near trivial fraction, $\sim 10^{-3}$ of the flux producing the magnetic jets. The galactic dynamo then need only maintain, (not generate by many e-folds), its initial trapped seed field. Classically an $\alpha \Omega$-dynamo in the galactic disk can not make nearly enough revolutions (only $<100$ turns in a Hubble time) needed to exponentiate by many e-folds to the final galactic fleld of $\sim 6 \times 10^{-6} \mathrm{G}$. The presumed starting, Biermann battery level seed fields are $\lesssim 10^{-20} \mathrm{G}--$ putatively from polarization currents from the dynamics at initial structure formation after de-coupling.

4) Faraday Rotation measures and Synchrotron radiation maps in radio lobe sources confirm the existence of magnetic fields. These fields are then fundamental to the mechanism of the radio lobe emission, which, in addition, requires a comparable energy density in ultra-relativistic electrons.

Faraday rotation measures the product of (the magnetic field aligned with the observer) $\mathrm{x}$ (the ambient electron density). Hence, when the field alters direction, the rotation measures may become confused. Fortunately the synchrotron polarization maps serve to establish a scale on which the field is coherent, namely, where the polarization vector is constant over a finite dimension. Furthermore the existence of polarized radio emission itself is almost uniquely indicative of synchrotron emission by energetic electrons in a magnetic field. The morphology of these emission vectors describe jets from the AGN where the direction oscillates back and forth $\sim 45$ degrees as a function of length as expected for a force-free helical field. Further in length the jet kinks and twists as expected for an unstable "pinch". In circumstances of AGN jets in galactic clusters where the electron density is large and measured independently by x-rays, the value and morphology of the Faraday rotation measures are consistent in magnitude and morphology (plus and minus change of flux direction across the symmetry axis) with a force-free helical jet from the AGN source and of strength consistent with the minimum energy model of synchrotron emission from the radio lobes. Theoretical models of these force-free helical jets predict much larger magnetic fields, $\sim \times 10^{8}$ at smaller radii, $\sim \times 10^{-8}$, but predicting emission is difficult because the ambient plasma is most likely excluded by the strong fields themselves.

5) The equi-partition between accelerated non-thermal electron energy, and magnetic field energy implies the direct acceleration of electrons and ions without an intermediate step of converting the magnetic energy to heat through $\eta J^{2}$ through classical reconnection.

This is because the magnitude of magnetic energy already challenges the upper limits of feasible mechanisms, and major heat losses before acceleration would create a highly visible plasma and a subsequent highly inefficient conversion of thermal plasma energy into a highly relativistic particle spectrum. This appears unlikely unless the thermal energy creates a strong shock wave in the IGM. There appears no evidence for such a shock in the ultra low density, $5 \times 10^{-6} / \mathrm{cm}^{3}$, of the IGM, and to make such a relativistic shock with a relativistic fluid would require a magnetic field of $B_{\text {shock }} \simeq 2 \times 10^{-5} \mathrm{G}$, which also would require the radio lobes expanding to $10 \mathrm{Mpc}$ in $10^{8} \mathrm{y}$. Hence we must find an alternate, efficient mechanism to convert the free energy of Force-Free twisted magnetic fields into rapid, direct acceleration of the current carriers comprising the current, $J_{\|}$, that supports the helical field. Instead we have suggested a unique mechanism to preferentially accelerate the current carriers, which requires the effect of starving the helical field of current carriers so that the remaining current carriers must be accelerated to a limiting velocity $c$, in order to carry the current. Otherwise the current will be reduced and $d B / d t$ will create a greater electric field, further accelerating the remaining current carriers. The 
result of current carrier starvation is the conversion of magnetic flux into electric field and consequential acceleration of the remaining current carrying particles.

The ultra strong gravity near the event horizon naturally creates current carrier starvation at the innermost boundary. An additional loss of accelerated particles occurs by random-walk diffusion, along the weakly tangled lines of force out of the helical field from weak instabilities on the ion plasma scale and furthermore "down" the field gradient. The return of ambient plasma or previously accelerated particles is similarly inhibited by the inverse, positive field gradient. The combination of diffusive instabilities and field gradient inhibition naturally maintains the current carrier starvation condition. These run-away, accelerated current carriers of the current carrier-starved, all $J_{\|}$current, $\left(\sim 3 \times 10^{18}\right.$ amperes) are then the accelerated electrons $\left(\Gamma \simeq 10^{4}\right)$ of the radio lobe emission due to curvature radiation and truncated in energy by the electron radio emission itself. Similarly, as in our galaxy one expects the ions to be accelerated preferentially because of much smaller $\propto\left(m_{e} / m_{p}\right)^{2}$ radiation loss, leading to the cosmic rays of the IGM.

6) The radiation spectrum from the accelerated electron current carriers of the ForceFree fields.

These accelerated electrons, $\Gamma \simeq 10^{4}$, lead to the synchrotron radio spectrum from radio lobes. In the stronger magnetic fields of the jet at smaller radii, $B \propto 1 / r$, these same accelerated electrons are a possible source of the polarized optical and possibly selfCompton x-rays from the synchrotron emission in the helical fields of the pinch or jets in the much stronger field at small radii. Finally x-rays from Compton collisions of these accelerated high energy electrons of the radio lobes with the CMB photons are expected, which have been interpreted from the morphology, spectrum, and x-ray luminosities.

7) Galaxy cluster magnetic fields from the central $S M B H$

The extreme motions within the cluster due to galaxy mergers with the cluster are predicted to amplify magnetic fields in the cluster by $\sim \times 10$ by the semi-coherent motions of ionized plasma. The higher density, $\sim \times 10^{3}$ of the cluster plasma compared to the IGM affords higher resolution Faraday rotation images of magnetic jets, intra-cluster radio lobe voids of mass density, and mergers. The PdV energies of the voids or bubbles within the cluster are comparable to the central AGN energy.

\section{The Total Energy: Radio Lobes, Relativistic Electrons \& Ions}

The largest source of magnetic energy and magnetic flux in the universe is that of the giant radio lobes of the FRII radio galaxies. The evidence for this is the interpretation of the radio flux in a frequency band, $\sim 1-10 \times 10^{9} \mathrm{~Hz}$ and size interpreted from distance and angular size. It was pointed out over 50 years ago (Burbridge, 1956) that a single AGN, "quasar" must release a very large magnetic energy, up to $\sim 10^{61}$ ergs, and that gravitational energy is the only feasible source (Hoyle et al. 1964, Burbidge \& Burbidge, 1965 and 1968). At that time the existence of a central massive black hole, (SMBH), of $10^{8} M_{\odot}$ at the center, $\left(\sim 3 \times 10^{-4}\right.$ of the exact center $)$ of every flat, spiral galaxy was unknown. Now the confirmed existence of such awesome objects makes these early physical deductions seem prescient.

The interpretation is based upon the observed luminosity in the radio, the size, and the observation of polarization, coherent over large fractions of the physical extent of the emission, for example M87, (Owen et al., 1989). Only synchrotron emission from relativistic electrons in a magnetic field seems feasible. The minimum total energy occurs when the magnetic and electron energies are comparable, $\sim 10^{61}$ ergs. Similarly when the size is estimated, a minimum total energy occurs for a given luminosity leading to 
magnetic fields of $\sim 10 \mu \mathrm{G}$ and a spectrum of relativistic electrons of mean energy of $\Gamma m_{e} c^{2}$, with $\Gamma \sim 10^{4},(50 \mathrm{Gev})$.

How could one accelerate so many electrons, $N_{e} \sim 10^{63}$, to such energies within an equal energy magnetic field? What other evidence exists for such an extreme result? Is it a ubiquitous phenomenon in the universe? Does the magnetic flux last long enough to "magnetize" the universe and affect the distribution of baryonic matter? or does the flux leak to the voids and reconnect in relatively short times, $\sim 10^{8}$ years?

Some years ago we, (Kronberg et al., 2001), asked this question and analyzed some 100 extra galactic radio lobes, (70 giants, $>0.6 \mathrm{Mpc}$, and $\sim 30$ Cluster-imbedded lobes ) finding a distribution in sizes, luminosity, and energy, using the formulation of (Pacholczyk, 1970) for analyzing the minimum energy. The result is that there are two distributions of radio lobes, one in the IGM and the other within galaxy clusters. The highest energy lobes are in the IGM with energies up to $\sim 10^{61}$ ergs. However, the radio lobes within galaxy clusters are less in energy by $\sim 1 / 100$. This large energy difference is understood by the existence of the x-ray bubbles in the cluster medium (Fabian et al., 2003). Since the pressure in the inter cluster medium, ICM, is $\sim \times 10^{6}$ greater than the IGM, it is a wonder that there is similar emission at all. Nevertheless a simulation of these bubbles by the injection of large helical flux from a central source, (presumably by the AGN dynamo and accretion disk) produces just these voids or bubbles in the ICM and requires the same extreme energy, $10^{61}$ ergs to make the voids in the ICM. (Diehl et al., 2008).

If this magnetic energy is in near equi-partition with relativistic electron energy then these highly relativistic electrons, $\Gamma \sim 10^{4}$, should interact with the microwave background, scattering x-rays from the CMB. This is exactly what is seen in the x-rays, 1 to $5 \mathrm{Kev}$ as mapped by Chandra, Croston et al. (2005). Thus if the size, luminosity, and relativistic electron spectrum fit the equi-partition model including the Comptonized $\mathrm{x}$-rays, then the final convincing proof is evidence of the magnetic fields as seen first by polarization maps of the lobe emission itself, (Kronberg et al., 1986), and secondly by Faraday rotation of polarized background sources. Fortunately similar more distance sources supply just such a probe and numerous observations of the characteristic rotation of the plane of polarization vs. frequency have been made, indicative of fields up to the equi-partition value of $\sim 10^{-5} \mathrm{G}$, Kronberg 1986 . The possibility of magnetic fields over much larger dimensions is demonstrated in the glow around the Coma cluster. (Kronberg et al., 2008).

Unfortunately both the model of the production mechanism of the fields and the plasma physics of the surrounding medium conspire to reduce the magnitude of the observed Faraday rotation signal. The winding of the dynamo flux by the AGN accretion disk leads to the averaging of many turns or reversals of the flux and hence reduces the Faraday rotation signal. In addition the likely background plasma density is reduced in the making the lobes as seen in the x-ray bubbles in clusters thereby reducing the expected Faraday rotation signal from within the lobes. Fortunately both these effects conspire to expect the largest rotation signal at largest radius, because the exclusion of plasma, ambient density, should be least at large radius just where the coherence of the rotation measure should be largest. In a few cases, such as seeing a helical structure edge on and within the higher density of the ICM, larger fields are confirmed, consistent with equi-partition of electron energy and magnetic field energy.

Combined this is awesome consistency of the model and therefore evidence for magnetic fields in the IGM on a scale of inter galactic structure, the spacing between galaxies and their organization into filaments. If $10^{61} \mathrm{ergs}$ of magnetic energy is distributed within the volume defined by the mean galaxy spacing, $\sim 1 \mathrm{Mpc}$ in the filaments, then assuming every galaxy, had at the time of its formation, an engine producing such flux, then the 
mean field becomes $\left\langle B>\sim 1.6 \times 10^{-6} \mathrm{G}\right.$. This field is larger by $\sim \times 10^{2}$ than the field as interpreted from Faraday rotation measurements of distant polarized sources. However, the possible multiple reversal averaging, the bubble reduction of ambient density, and finally the dissipation of magnetic flux and energy by reconnection or acceleration remain open questions. We believe the dissipation of magnetic flux actually occurs by acceleration rather than reconnection, because if the energy were converted first into heat by reconnection, then the IGM would be heated to $\simeq 10^{4} \mathrm{ev}$, and the radio emission would be missing from the kev electrons. We say heat, because current reconnection research leads to shocks and thus heat in the reconnection zone whereas we are suggesting a semi-coherent process where an $E_{\|}$leads to the direct acceleration of the charged current carriers.

\section{Extra-Galactic Cosmic Rays}

Within the galaxy, the "milky way", cosmic rays of energy density $\sim 1 \mathrm{eV} / \mathrm{cm}^{3}, 1.6 \times$ $10^{-12} \mathrm{ergs} / \mathrm{cm}^{3}$, are in equi-partition with the galactic magnetic field, $B_{\text {gal }} \simeq 6 \times 10^{-6} \mathrm{G}$. This equality suggests that the magnetic energy is the source of the particle energy or at least that the magnetic energy density determines the probability of the escape of accelerated cosmic rays from the galaxy. In either case the two are likely tightly dependent on each other. In our view extragalactic cosmic rays are likely to follow a similar acceleration mechanism and similar escape physics, that is escape from the filaments to the voids. Based on these assumptions, (Colgate \& Li, 2004) analyzed the likely extra galactic cosmic ray spectrum and energy density starting with the flux and energy of UHEs and extrapolating to lower energy, $m_{p} c^{2}$, and concluded that the energy density of the extra galactic CR flux may be $\sim 1 / 200$ of the galactic flux. This ratio is the likely ratio of the infall pressure to the filaments from the voids that is necessary to double the filament mass in a Hubble time as compared to the infall pressure from the halo to the galaxy necessary to double the galaxy gas mass in a Hubble time. The magnetic field and particle pressure are then in equilibrium with the confining pressure of the in-falling, accreting matter. The total energy in extra galactic CRs per galaxy spacing volume is then $\sim 10^{60}$ ergs, somewhat less than what one might expect from the typical AGN-radio lobe. However, some leakage to the voids is expected at the highest energies in a Hubble time and furthermore the SMBH of our Galaxy is significantly smaller than the average implying a smaller initial AGN and radio lobe.

Thus the interpretation of immense extra galactic magnetic fields and equal immense energy in relativistic ions and electrons is supported by the polarization maps, radio luminosity, size, Faraday rotation, luminosity in x-rays, CRs, and CR energy. In turn this is consistent with the synchrotron emission of the equi-partition model of radio lobes.

\section{The Origin of the Magnetic Flux and Energy}

The magnetic flux of radio lobes is of order (area $\mathrm{x}$ field) or $\sim 10^{42} \mathrm{G}-\mathrm{cm}^{2}$. This is $\times 10^{3}$ greater than the flux of the galactic magnetic field, yet to create a galactic dynamo that could make this much flux starting from the "Bierman battery" level of $\sim 10^{-20} \mathrm{G}$ of the galaxy, seemed nearly impossible, (Kulsrud, R. M. \& Zweibel, E. G., 2008). Hence we agree with their suggestion that the galactic flux was "seeded" from the AGN accretion disk dynamo before the galaxy formed and then only maintained by the galactic dynamo in pressure equilibrium with the in-fall pressure from the halo. Here the trapping of only 
$\sim 10^{-3}$ of the helical force-free jet flux within the galaxy is required to "seed" the galactic flux.

Nearly universally the $\alpha \Omega$ dynamo (Parker, 1955; Parker, 1979) has been the accepted mechanism for converting kinetic energy into magnetic energy, yet galactic motions alone are inadequate, because the galaxy makes only $\sim 100$ turns, the $\Omega$-motion, in a Hubble time and the source of the helicity, the $\alpha$-motion by supernova remnant bubbles occurs on a scale too large for the flux to merge in a Hubble time using laminar flow. Thus the generation of magnetic flux by the galaxy seems frustrated on both levels of the $\alpha \Omega$ dynamo. These problems were addressed in (Pariev, Colgate, \& Finn, 2007; Pariev, Colgate, 2007) where it was recognized and calculated that the accretion disk forming the SMBH and AGN was a unique circumstance where the disk matter makes many turns, $\sim 10^{11}$ during its life time. Consequently the dynamo gain can be so large that the question of seed field becomes moot. Equally important was the possibility that an expanding plume driven axially outwards from the disk in a rotating frame had the unique property of a 1/4 turn of rotation before merging back with the disk and thus for creating the helicity necessary for an astrophysical dynamo. Such a dynamo was simulated and analyzed by axial vector simulation, mean field theory, and flux conversion, (Pariev, Colgate, \& Finn, 2007). The formation of the large scale generation of magnetic flux in the accretion disk forming the SMBH of an AGN was a natural prediction. Here a small mass fraction of the disk, $\sim<10^{-5}$, was assumed to be in stars in random orbits around the SMBH. This mass fraction of stars is less than the metalicity, usually assumed in pre-galactic stars, $\sim 10^{-3}$. These stars collide with the disk creating buoyancy driven plumes, and thus the helicity necessary for the dynamo. Furthermore the $\alpha_{T}$-viscosity turbulence gives rise to the necessary flux diffusion so that flux can merge, link, or multiply in a finite number of turns. We believe that this is a unique solution to this problem, of creating the immense, large scale magnetic flux and energy of the ISM. This is because:

1) Half the free energy of accretion, $\sim 10^{62}$ ergs can potentially be converted to magnetic energy.

2) The total number of turns at $3 r_{g}$ is $N_{T} \sim 10^{11}$ in the formation time, $10^{8}$ years of the SMBH and thus the question of seed field moot.

3) The helicity produced poloidal flux is in the same direction and $1 / 4$ turn for every plume and thus the average poloidal flux is the arithmetic sum of the flux from each plume, not the statistical vector average as might be expected from turbulence alone.

4) When the plume falls back to the disk, the plume matter and imbedded flux mixes and diffuses with the turbulent diffusion coefficient of the plume-disk mixing turbulence, faster than the $\alpha_{T}$-viscosity without angular momentum stabilization, thus allowing the small loop of poloidal flux to rapidly merge with others coherently. All loops are in the same direction, thus creating the large scale poloidal flux of the dynamo.

5) The mass density of the plumes is sufficient so that the moment of inertia of the plume will twist the fields up to the emission limit, or Eddington limit, $B_{r g} \simeq 3 \times 10^{4} \mathrm{G}$.

6) The diffusive separation of circular poloidal flux foot prints by the $\alpha_{T}$-viscosity in the disk allows a large differential winding of the Keplerian motion between the two cylindrical foot-prints, an inner one of flux leaving the disk and an outer one of flux returning to the disk. In the conducting medium of the IGM this leads to continuous differential winding, $\sim 10^{11}$ turns of the flux surfaces, producing the near force-free helix of the jets and radio lobes. (Li, H. et al., 2001) 


\section{Experimental Dynamos}

There have been two liquid sodium dynamo experiments with positive results, both with flows that are turbulence-constrained by rigid walls. In three recent experiments using counter rotating converging flow, large turbulence in the unconstrained shearing mid-plane flow reduced the $\Omega$-gain to $\simeq<\times 2$. In the New Mexico sodium experiment, where the flow is partially stabilized by angular momentum, Couette flow, the $\Omega$-gain increased to $\simeq \times 8$.

\section{Summary}

The evidence for large scale and large energy magnetic fields with equi-partition with accelerated particles in the IGM is convincing. An $\alpha \Omega$ dynamo in the accretion disk of the SMBH creates poloidal field, (in stars as well). The winding of this poloidal flux by the accretion disk into a force-free helical jet is the likely explanation of the AGN jet, the radio lobes, and accelerated particles.

\section{Acknowledgements}

The experimental work has been supported by Howard Beckley, David Westpfahl, Jaihe $\mathrm{Si}$, Joe Martinque, and a dozen undergraduate students at NMTechr. Vladimir Pariev first calculated the plume-driven $\alpha \Omega$ dynamo. The liquid sodium dynamo experiment has been supported by DOE through the MOU between Univ. of Calif. at Los Alamos Nation Lab and New Mexico Institute of Mining and Technology. The theory and experiment have both been supported though the LDRD funding by the DOE and LANS.

\section{References}

Burbidge, G. R. 1956, Ap.J., 124, 416

Burbidge, G. R. 1958, Ap.J., 129, 849

Burbidge, G. R. \& Burbidge, E. M. 1965, The Structure and Evolution of Galaxies, Proc. $13^{\text {th }}$ (Solvay) Conf. on Physics, Bruxelles, New York: Interscience, Wiley, 137

Chandrasekhar, S., 1960, Proc. Natl. Acad. Sci., 46, 253

Colgate, S. A. \& Li, Hui, 2004, Reconnection of Force-Free Fields, ed. Guenter Sigl and Murat Boratav in The French Academy of Sciences, Comptes rendus - Physique, C.R. Physique 5431 (astro-ph/0509054)

Croston, J. H., Hardcastle, M. J., Harris, D. E., Belsole, E. Birkinshaw, M., \& Worrall, D. M. 2005, Ap.J. 626, 733

Diehl, S., Li, Hui, Fryer, C., \& Rafferty, D. 2008, Ap.J. 687, 173

Fabian, A. C., Sanders,J. S., Crawford, C. S., Conselice, C. J., Gallagher III J. S., \& Wyse, R. F. G., 2003 Mon. Not. R. Astron. Soc. 344, L48 (2003)

Hoyle, F., Fowler, W. A., Burbidge, G. R., \& Burbidge E. M. 1964, Ap.J., 139, 909

Kronberg, P. P. Wielebinski, l. R., \& Graham, D. A. 1986, Astron. Astrophys. 169, 63

Kronberg, P. P., Dufton, Q., Li, H., \& Colgate, S. A. 2001, Ap.J., 560, 178

Kronberg, P. P., Kothes, R., Salter, C., \& Perillat, P. 2008, Ap.J., 659.267

Kulsrud, R. M. \& Zweibel, E. G. 2008, Reports on Progress in Physics, 71, 046901 (2008).

Li, H., Lovelace, R. V. E., Finn, J. M., \& Colgate, S. A. Ap.J., 561, 915

Mestel, L. 1999, Stellar Magnetism. (Oxford: Clarendon)

Owen, F. N., Hardee, P. E., \& Cornwell, T. J. 1989, Ap.J., 340698

Pacholczyk, A. G. 1970, Radio Astrophysics (San Francisco: Freeman)

Pariev, I. V., Colgate, S. A., \& Finn, J. M. 2007, Ap.J., 658, 114.

Pariev, I. V., Colgate, S. A., \& Finn, J. M. 2007, Ap.J., 658, 129.

Parker, E. N. 1955, Ap.J. 121, 29

Parker, E. N. 1979, Cosmical Magnetic Fields, their Origin and their Activity. (Oxford: Claredon) 